Research Paper

\title{
Serum Prealbumin is Negatively Associated with Survival in Hepatocellular Carcinoma Patients after Hepatic Resection
}

\author{
Ying-Yang Liao"\#, Chun-Lan Teng2\#, Ning-Fu Peng2,3\#, Rong-Rong Jia2,3, Jing Cui², Kang Chen², Liang \\ $\mathrm{Ma}^{2,3}$, Bang-De Xiang ${ }^{2,3}$, Jian-Hong Zhong ${ }^{2,3}$, Le-Qun Li ${ }^{2,3}$ \\ 1. Department of Nutrition, Affiliated Tumor Hospital of Guangxi Medical University, Nanning, China \\ 2. Department of Hepatobiliary Surgery, Affiliated Tumor Hospital of Guangxi Medical University, Nanning, China \\ 3. Guangxi Liver Cancer Diagnosis and Treatment Engineering and Technology Research Center, Nanning, China \\ \#These authors contributed equally to this work.
}

$\square$ Corresponding authors: Jian-Hong Zhong and Bang-De Xiang, Department of Hepatobiliary Surgery, Affiliated Tumor Hospital of Guangxi Medical University, HeDi Rd \#71, Nanning 530021, the People's Republic of China. Email: zhongjianhong@gxmu.edu.cn or zhongjianhong66@163.com (J.-H.Z); xiangbangde@gxmu.edu.cn (B.-D.X); Telephone: +86 771533 0855; Fax: +86 7715312000.

(c) Ivyspring International Publisher. This is an open access article distributed under the terms of the Creative Commons Attribution (CC BY-NC) license (https://creativecommons.org/licenses/by-nc/4.0/). See http://ivyspring.com/terms for full terms and conditions.

Received: 2018.10.23; Accepted: 2019.05.09; Published: 2019.06.02

\begin{abstract}
Objective: Low serum prealbumin levels are associated with poor prognoses in some type of cancers. However, the role of prealbumin in patients with hepatocellular carcinoma (HCC) is unknown. The present study aimed to investigate the role of serum prealbumin levels in long-term survival for HCC patients after hepatic resection.

Methods: HCC patients who underwent hepatic resection from June 2007 to December 2015 were retrospectively analyzed in a tertiary liver center. Patients were classified as having normal or reduced serum prealbumin based on a cut-off value of $200 \mathrm{mg} / \mathrm{L}$. Overall survival and recurrence rate were analyzed between groups. Propensity score analysis was used to reduce bias due to other patient differences at baseline.

Results: A total of $1349 \mathrm{HCC}$ patients who underwent hepatic resection were enrolled on this study, including 1168 (86.6\%) male and 181 (13.4\%) female. Patients with normal serum prealbumin had significantly higher overall survival than those with reduced serum prealbumin $(P<0.001)$. Similar findings were observed after propensity analysis and subgroup analysis based on liver cirrhosis. Moreover, patients with normal serum prealbumin had a significantly lower recurrence rate than those with reduced serum prealbumin $(P<0.001)$.

Conclusions: Low preoperative level of serum prealbumin is associated with poor long-term survival in patients with $\mathrm{HCC}$ after hepatic resection. Low serum prealbumin may be a marker to identify patients at high risk of poor prognosis after hepatic resection.
\end{abstract}

Key words: humans; clinical study; prognosis

\section{Introduction}

Hepatocellular carcinoma (HCC) is the third leading cause of cancer-related death worldwide. ${ }^{1}$ The incidence of HCC and associated mortality are anticipated to grow in the next decade, particularly in western countries. ${ }^{1} \mathrm{HCC}$ is difficult to treat because its treatments depend on not only tumor stage, but also liver function. Based on official guidelines and clinical practices, ${ }^{2,3}$ hepatic resection is the main treatment for HCC. However, the success of hepatic resection is largely dependent on the health of the liver, especially the degree of liver cirrhosis, which also affects long-term overall survival after surgery. ${ }^{4}$ 
Nutritional status has been reported to predict perioperative morbidity and mortality after hepatic resection in HCC patients. 5,6 Serum albumin level has been routinely used in clinical practice as a surrogate marker to evaluate nutritional status and liver function. Serum albumin concentration is used as an independent mortality risk predictor in a broad range of clinical and research settings. ${ }^{7}, 8$ However, measurement of serum albumin can be inaccurate because the level of serum albumin can be affected by blood transfusion or other factors. Serum prealbumin provides an alternative because its level is less affected by liver diseases, even though it is synthesized by the liver. ${ }^{9}$ Moreover, serum prealbumin level is a good indicator of nutritional status. ${ }^{9}$ A few recent studies reported that low preoperative prealbumin level is a negative independent prognostic factor for cancer-specific survival.10, 11 However, the relationship between preoperative serum prealbumin level and long-term prognosis in patients with HCC after hepatic resection has not been fully elucidated. Therefore, this retrospective study aimed to investigate the role of preoperative serum prealbumin level on the long-term survival in HCC patients.

\section{Patients and Methods}

Medical records were retrospectively examined to identify patients with confirmed hepatic resection and post-operative HCC diagnosis between June 2007 to December 2015 at the Affiliated Tumor Hospital of Guangxi Medical University. Patients were included in this study if they (a) were admitted for initial HCC treatment at our hospital; (b) had no treatment prior to hepatic resection and no other malignancies; (c) were followed-up at our hospital for more than 3 months; (d) had complete clinical data, including serum albumin and prealbumin. This retrospective study was approved by the Ethics Committee of the Hospital.

Hepatic resection and follow-up procedures were described in detail previously. ${ }^{12,13}$ Clinical stage of HCC was determined according to the guidelines of the European Association for the Study of the Liver Clinical Practice. ${ }^{2}$ Liver cirrhosis was confirmed by histopathological examination. Follow-up investigations consisted of blood testsfor liver function and alpha-fetoprotein levels, abdominal ultrasonography, computed tomography, or magnetic resonance imaging. Survival time was defined as the interval (in months) from the date of hepatic resection to the date of last follow-up (31 July 2018) or death. Serum prealbumin was measured as part of liver function testing after overnight fasting at 6:00-7:00 a.m. on the second morning of hospitalization.
Normal level of serum prealbumin was defined as at $200 \mathrm{mg} / \mathrm{L}$ or higher, so this value was used to classify patients as having "reduced" or "normal" levels. This normal level is based on our hospital's experience. Liver function was tested with ADVIA $^{\circledR} 2400$ Chemistry System (Siemens Healthcare Diagnostics Inc).

Data were analyzed using SPSS 17.0 (IBM, Armonk, NY, USA). Categorical data were presented as frequency and percentage (\%). In propensity score analysis, differences between patients with reducedor normal prealbumin were assessed for significance using Pearson chi-square test or Fisher's exact test. Survival curves were estimated using Kaplan-Meier method and compared by log rank test. Multivariate analyses were performed using Cox regression proportional hazards model to identify potential risk factors associated with mortality. Subgroup analysis was performed to examine a potential relationship between serum prealbumin level and overall survival for patients with or without liver cirrhosis. Spearman rank-order correlation analysis was used to identify the correlation between albumin and prealbumin levels. Differences were considered significant in all statistical tests when $\mathrm{P}<0.05$ (two-tailed).

In order to reduce bias in patient selection, propensity score analysis was carried out using logistic regression to create propensity scores for reduced and normal serum prealbumin groups in an observational database. Logistic regression was applied to clinical variables when they differed significantly between reduced and normal groups and propensity scores were generated along a continuous range from 0 to 1 . Propensity score model was then used to provide a one-to-one nearest-neighbor match between reduced and normal groups.

\section{Results}

Between June 2007 and December 2015, 1780 patients with HCC underwent hepatic resection at our tertiary liver center. Based on inclusion criteria, 1349 $(75.8 \%)$ patients were included in this study, in which $1168(86.6 \%)$ were male and 181 (13.4\%) were female. Among 1349 patients, 649 had normal serum prealbumin levels $(\geq 200 \mathrm{mg} / \mathrm{L})$ and 700 had reduced levels $(<200 \mathrm{mg} / \mathrm{L})$.

Preoperative prealbumin level correlated significantly with age, gender, HCC family history, macrovascular invasion, tumor size, tumor stage, hepatitis B surface antigen, albumin, total bilirubin, Child-Pugh class, and alpha-fetoprotein (Table 1). Preoperative prealbumin level did not, however, correlate with liver cirrhosis, tumor number, tumor capsule or alanine transaminase (Table 1). Spearman correlation analysis found positive correlation 
between preoperative albumin and prealbumin levels $(\mathrm{R}=0.494, P<0.001$; Figure $1 \mathrm{~A})$. Moreover, patients with liver cirrhosis had significantly lower preoperative prealbumin levelsthan those without $(P$ $=0.002$; Figure 1B).

Median length of patient follow-up was 45.3 months. Patients with serum prealbumin $\geq 200 \mathrm{mg} / \mathrm{L}$ showed significantly higher rates of disease-free survival than those with prealbumin $<200 \mathrm{mg} / \mathrm{L}$ at 1 year ( $65 \%$ vs. $49 \%), 3$ years ( $35 \%$ vs. $22 \%)$, and 5 years (33\% vs. $6 \%)(P<0.001$, Figure 2$)$. Moreover, overall survival rates were significantly higher among patients with serum prealbumin $\geq 200 \mathrm{mg} / \mathrm{L}$ than among those with prealbumin $<200 \mathrm{mg} / \mathrm{L}$ at 1year $(89 \%$ vs. $81 \%)$, 3years $(72 \%$ vs. $59 \%)$, and 5years $(62 \%$ vs. $50 \%)(P<0.001$, Figure $3 \mathrm{~A})$. Propensity score analysis showed similar results $(P<0.001$, Figure $3 \mathrm{~B})$.

Since levels of serum albumin and prealbumin may reflect the degree of liver cirrhosis, subgroup analysis was performed depending on whether patients had liver cirrhosis or not. We found that reduced prealbumin level was associated with worse overall survival than normal prealbumin, regardless of whether patients had liver cirrhosis (Figure 3C) or not (Figure 3D).

Table 1. Relationships between preoperative prealbumin levels and clinicopathological factors.

\begin{tabular}{|c|c|c|c|c|c|c|c|}
\hline \multirow[t]{2}{*}{ Factor } & \multirow[t]{2}{*}{$\mathrm{N}=1349(\%)$} & \multicolumn{3}{|l|}{ Total population } & \multicolumn{3}{|l|}{ After propensity scoring } \\
\hline & & $\mathrm{pALB} \geq 200 \mathrm{mg} / \mathrm{L}(n=649)$ & $\mathrm{pALB}<200 \mathrm{mg} / \mathrm{L}(n=700)$ & $P$ & $\mathrm{pALB} \geq 200 \mathrm{mg} / \mathrm{L}(n=381)$ & $\mathrm{pALB}<200 \mathrm{mg} / \mathrm{L}(n=381)$ & $P$ \\
\hline \multicolumn{8}{|l|}{ Age, year } \\
\hline$>60$ & $228(16.9)$ & $92(14.2)$ & $136(19.4)$ & 0.011 & $63(16.5)$ & $51(13.4)$ & 0.264 \\
\hline$\leq 60$ & $1121(83.1)$ & $557(85.8)$ & $564(80.6)$ & & $318(83.5)$ & $330(86.6)$ & \\
\hline \multicolumn{8}{|l|}{ Gender } \\
\hline Male & $1169(86.7)$ & $595(91.7)$ & $574(82.0)$ & $<0.001$ & $340(89.2)$ & $339(89.0)$ & 1.000 \\
\hline Female & $180(13.3)$ & $54(8.3)$ & $126(18.0)$ & & $41(10.8)$ & $42(11.0)$ & \\
\hline \multicolumn{8}{|c|}{ Hepatocellular carcinoma family history } \\
\hline Present & $234(17.3)$ & $127(19.6)$ & $107(15.3)$ & 0.044 & $42(11)$ & $53(13.9)$ & 0.273 \\
\hline Absent & $1115(82.7)$ & $522(80.4)$ & $593(84.7)$ & & $339(89)$ & $328(86.1)$ & \\
\hline \multicolumn{8}{|l|}{ Cirrhosis } \\
\hline No & $597(44.3)$ & $298(45.9)$ & $299(42.7)$ & 0.249 & $161(42.3)$ & $156(40.9)$ & 0.769 \\
\hline Yes & $752(55.7)$ & $351(54.1)$ & $401(57.3)$ & & $220(57.7)$ & $225(59.1)$ & \\
\hline \multicolumn{8}{|c|}{ Macrovascular invasion } \\
\hline No & $1094(80.4)$ & $559(86.1)$ & $535(76.4)$ & $<0.001$ & $310(81.4)$ & $312(81.9)$ & 0.926 \\
\hline Yes & $255(19.6)$ & $90(13.9)$ & $165(23.6)$ & & $71(18.6)$ & $69(18.1)$ & \\
\hline \multicolumn{8}{|c|}{ Tumor number } \\
\hline$\leq 3$ & $1274(94.4)$ & $614(94.6)$ & $660(94.3)$ & 0.813 & $367(96.3)$ & $363(95.3)$ & 0.589 \\
\hline$>3$ & $75(5.6)$ & $35(5.4)$ & $40(5.7)$ & & $14(3.7)$ & $18(4.7)$ & \\
\hline \multicolumn{8}{|c|}{ Tumor size, $\mathrm{cm}$} \\
\hline$\leq 5$ & $533(39.5)$ & $326(50.2)$ & $207(29.6)$ & $<0.001$ & $141(37.0)$ & $145(38.1)$ & 0.822 \\
\hline$>5$ & $816(60.5)$ & $323(49.8)$ & $493(70.4)$ & & $240(63.0)$ & $236(61.9)$ & \\
\hline \multicolumn{8}{|c|}{ Barcelona Clinic Liver Cancer Stage } \\
\hline $0 / \mathrm{A}$ & $862(63.9)$ & $445(68.6)$ & $417(59.6)$ & $<0.001$ & $246(64.6)$ & $255(66.9)$ & 0.672 \\
\hline B & $214(15.9)$ & $108(16.6)$ & $106(15.1)$ & & $64(16.8)$ & $55(14.5)$ & \\
\hline $\mathrm{C}$ & $273(20.2)$ & $96(14.8)$ & $177(25.3)$ & & $71(18.6)$ & $71(18.6)$ & \\
\hline \multicolumn{8}{|c|}{ Tumor capsule } \\
\hline Complete & $889(65.9)$ & $437(67.3)$ & $452(64.6)$ & 0.301 & $248(65.1)$ & $248(65.1)$ & 1.000 \\
\hline Incomplete & $460(34.1)$ & $212(32.7)$ & $248(35.4)$ & & $133(34.9)$ & $133(34.9)$ & \\
\hline \multicolumn{8}{|c|}{ Hepatitis B surface antigen } \\
\hline Negative & $181(13.4)$ & $111(17.1)$ & $70(10.0)$ & $<0.001$ & $47(12.3)$ & $37(9.7)$ & 0.298 \\
\hline Positive & $1169(86.6)$ & $538(82.9)$ & $630(90.0)$ & & $334(87.7)$ & $344(90.3)$ & \\
\hline \multicolumn{8}{|c|}{ Albumin, g/L } \\
\hline$\leq 35$ & $121(9.0)$ & $11(1.7)$ & $110(15.7)$ & $<0.001$ & $9(2.4)$ & $11(2.9)$ & 0.821 \\
\hline$>35$ & $1228(91.0)$ & $638(98.3)$ & $590(84.3)$ & & $372(97.6)$ & $370(97.1)$ & \\
\hline \multicolumn{8}{|c|}{ Alanine transaminase, $\mathrm{U} / \mathrm{L}$} \\
\hline$\leq 40$ & $816(60.5)$ & $397(61.2)$ & 419 (59.9) & 0.656 & $229(60.1)$ & $226(59.3)$ & 0.883 \\
\hline$>40$ & $533(39.5)$ & $252(38.8)$ & $281(40.1)$ & & $152(39.9)$ & $155(40.7)$ & \\
\hline \multicolumn{8}{|c|}{ Total bilirubin, $\mu \mathrm{mol} / \mathrm{L}$} \\
\hline$\leq 21$ & $1220(90.4)$ & $607(93.5)$ & $613(87.6)$ & $<0.001$ & $353(92.7)$ & 350 (91.9) & 0.787 \\
\hline$>21$ & $129(9.6)$ & $42(6.5)$ & $87(12.4)$ & & $28(7.3)$ & $31(8.1)$ & \\
\hline \multicolumn{8}{|c|}{ Child-Pugh class } \\
\hline A & $1245(92.3)$ & $609(93.8)$ & $636(90.9)$ & $<0.001$ & $379(99.5)$ & $379(99.5)$ & 1.000 \\
\hline B & $104(7.7)$ & $40(6.2)$ & $64(9.1)$ & & $2(0.5)$ & $2(0.5)$ & \\
\hline \multicolumn{8}{|c|}{ Alpha fetoprotein, $\mathrm{ng} / \mathrm{mL}$} \\
\hline$\leq 400$ & $770(57.1)$ & $393(60.6)$ & $377(53.9)$ & 0.015 & $225(59.1)$ & $231(60.6)$ & 0.712 \\
\hline$>400$ & $578(42.9)$ & $256(39.4)$ & $322(46.1)$ & & $156(40.9)$ & $150(39.4)$ & \\
\hline
\end{tabular}

Data are $\mathrm{n}(\%)$. pALB, prealbumin. 

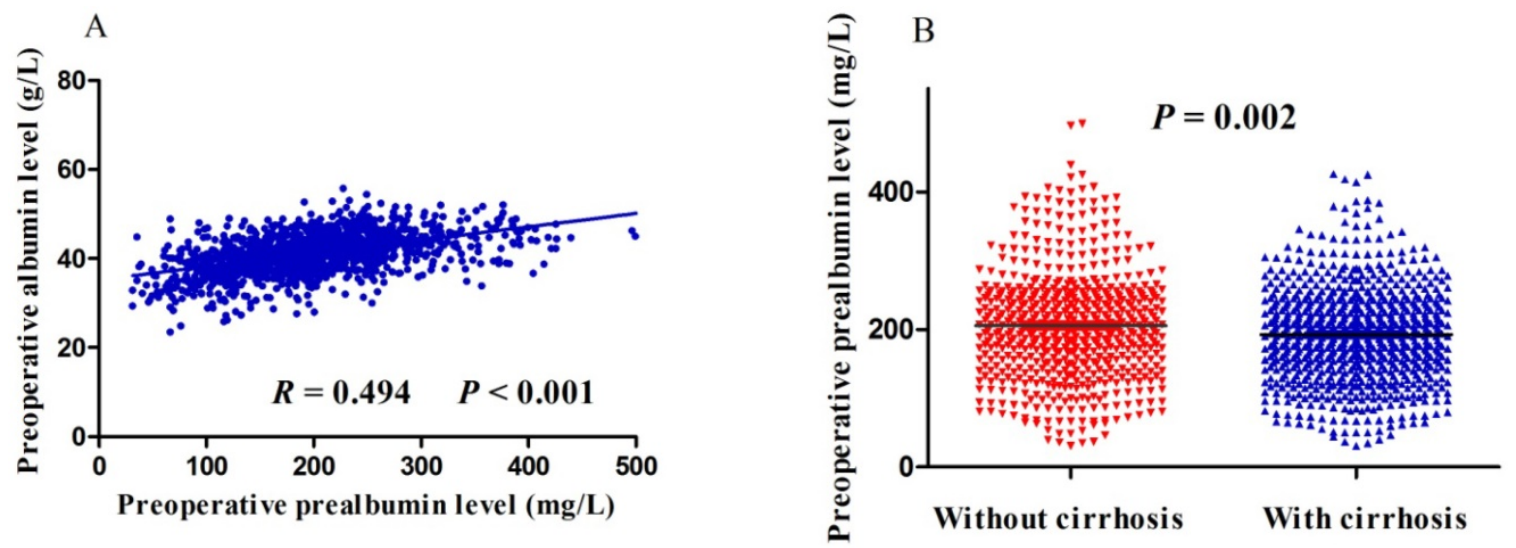

Figure 1. Scatter plots of (A) the relationship between preoperative levels of albumin and prealbumin, or (B) the difference in preoperative prealbumin levels between patients with or without liver cirrhosis.

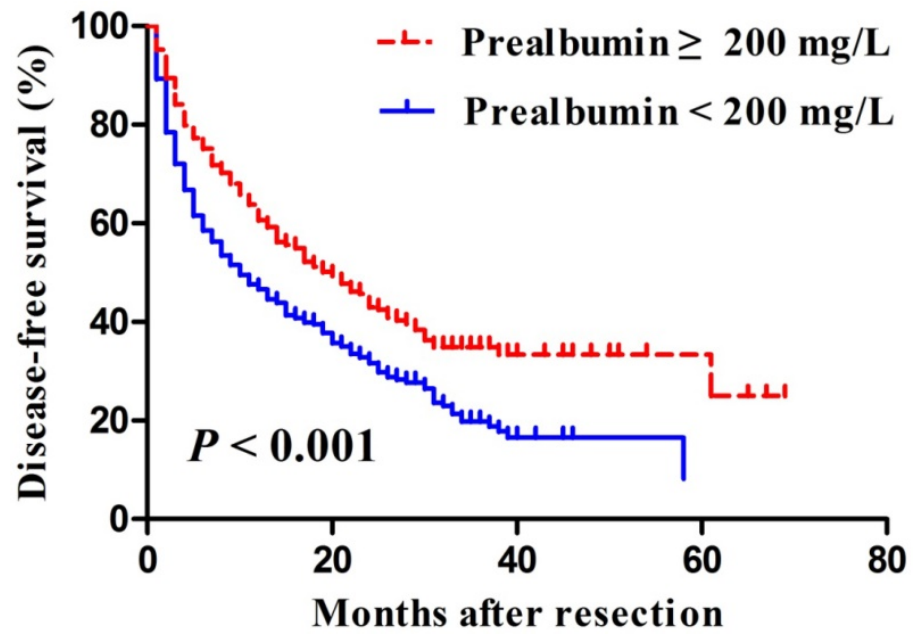

Figure 2. Kaplan-Meier curves of post-resection disease-free survival in hepatocellular carcinoma patients with a normal or reduced preoperative serum prealbumin, based on a cut-off value of $200 \mathrm{mg} / \mathrm{L}$.
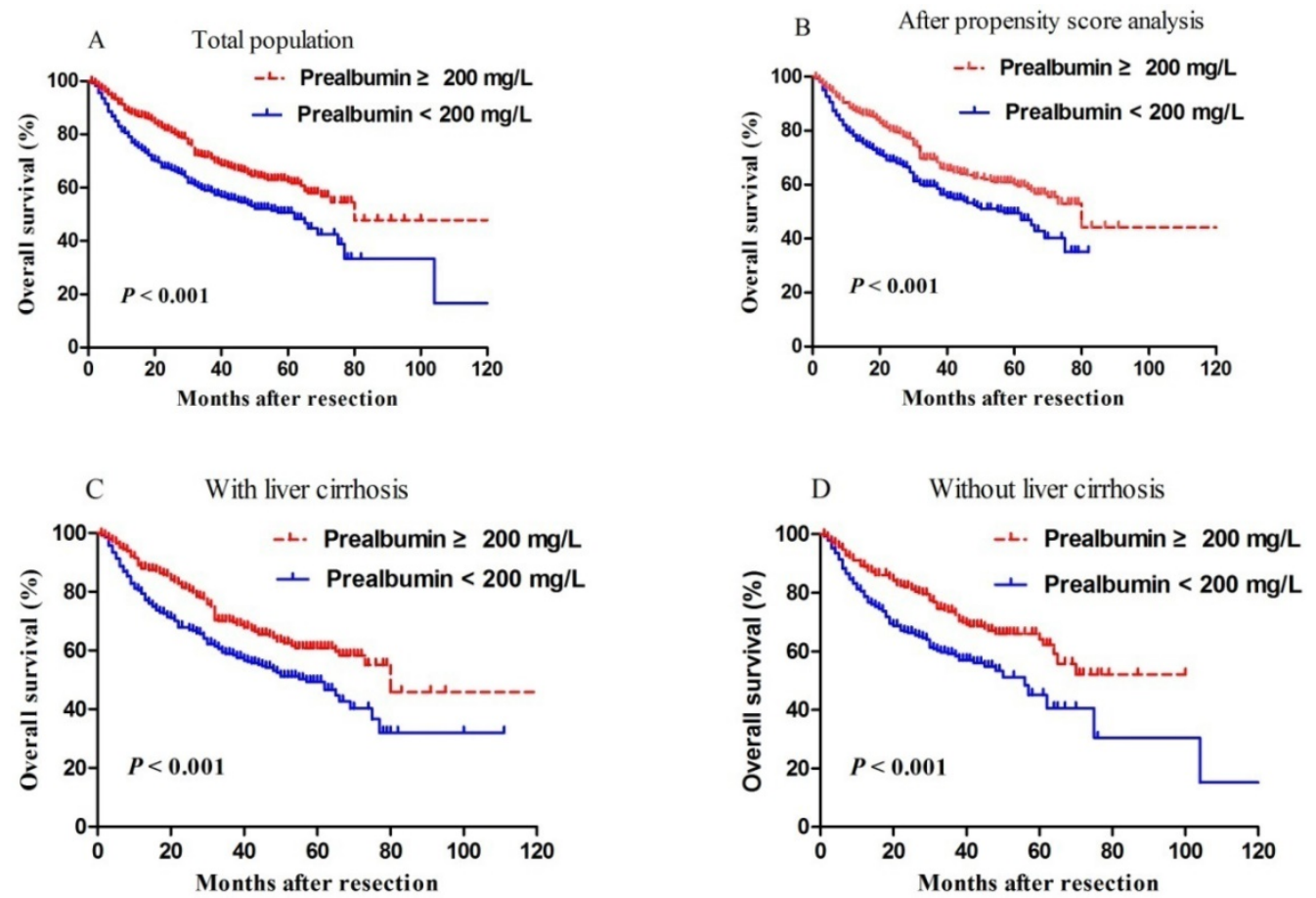

Figure 3. Kaplan-Meier curves of post-resection overall survival in (A) all patients, (B) propensity score-matched pairs of patients, (C) patients with cirrhosis or (D) patients without cirrhosis. In all cases, patients were stratified into those with normal or reduced preoperative levels of prealbumin, based on a cut-off value of $200 \mathrm{mg} / \mathrm{L}$. 


\section{Discussion}

In recent decades, many preoperative clinicopathologic variables were used to predict postoperative liver dysfunction, perioperative mortality, and long-term overall survival, such as serum markers of liver fibrosis, ${ }^{14}$ splenic thickness, ${ }^{15}$ neutrophil-to-lymphocyte ratio, ${ }^{16}$ Controlling Nutritional Status Score, ${ }^{5}$ body-mass index, ${ }^{17}$ and age $^{18}$. Estimation of hepatic functional reserve in the future remnant liver is critical for HCC patients before hepatic resection.

Both Child-Pugh and albumin-bilirubin scores ${ }^{8}$ 19 include albumin to predict hepatic functional reserve before surgery. However, the level of serum albumin can be affected by supplemental infusion of human serum albumin and blood transfusion. This exogenous albumin persists in the body a long time, which affects the accuracy of liver function estimation because the half-life of albumin is about 20 days. ${ }^{20}$ Therefore, many studies chose prealbumin as a sensitive marker to predict prognosis because the half-life of prealbumin is only 0.5 day..$^{21}$ Serum prealbumin level reflects liver protein synthesis and secretion. Therefore, measuring serum prealbumin levels can predict early liver function damage and prognosis of some diseases. ${ }^{22}$ For example, prealbumin levels have been used as a marker for predicting infectious complications after gastric surgery, ${ }^{23}$ the risk of recurrent colorectal cancer, ${ }^{24}$ early recurrence after curative pulmonary resection for non-small-cell lung cancer, ${ }^{25}$ and postoperative liver insufficiency in hepatic resection patients. ${ }^{26}$

The present study examined the ability of preoperative serum prealbumin level to predict overall and disease-free survival after liver resection in a large cohort of HCC patients in a tertiary liver center. We found that preoperative serum prealbumin level may be a useful marker for predicting long-term overall and disease-free survival, in the presence or absence of liver cirrhosis. These findings are consistent with previous studies.27, 28 A previous study used a cut-off value of $170 \mathrm{mg} / \mathrm{L}$ for preoperative prealbumin level compared to $200 \mathrm{mg} / \mathrm{L}$ in the present study. They found only $29 \%(437 / 1483)$ patients with reduced prealbumin level, lower than the $51.9 \%(700 / 1349)$ in the present study. ${ }^{28}$ Our previous small study used a cut-off value of $182 \mathrm{mg} / \mathrm{L}$ for preoperative prealbumin level. ${ }^{27}$ Other studies found that prealbumin level lower than $170 \mathrm{mg} / \mathrm{L}$ is an independent risk factor for hepatic insufficiency after hepatic resection. ${ }^{26,}{ }^{29}$ In other types of cancers, prealbumin level lower than $220 \mathrm{mg} / \mathrm{L}$ is associated with higher rate of recurrence and lower rate of survival in colorectal cancer. ${ }^{24}$ Prealbumin level of $<230 \mathrm{mg} / \mathrm{L}$ has been associated with greater risk of non-small cell carcinoma. ${ }^{25}$ Therefore, finding an appropriate prealbumin cut-off level for a particular type of cancer is critical for predicting tumor recurrence and patient survival.

A number of conditions are known to affect the level of prealbumin and/or albumin, including liver cirrhosis, hepatitis B/C virus, loss of compensatory liver function, and abnormal nutritional status. ${ }^{28}$ Liver cirrhosis is one of the decisive factors for liver function, which critically affects prognosis after hepatic resection. Our results showed that HCC patients with liver cirrhosis had lower prealbumin level, which was associated with poorer patient survival. These results support the idea that serum prealbumin level may predict pre-existing liver cirrhosis, which is critical for liver function and therefore prognosis in HCC patients after liver resection.

There are a few limitations in this study. The main drawback is the lack of internal or external validation. However, our previous study with small sample size used a cut-off value of $182 \mathrm{mg} / \mathrm{L}$ for preoperative prealbumin level found similar results. Moreover, other study from other province of China also found similar results. Second, patient prealbumin level groups were not assigned randomly. However, propensity score analysis was used in the present study to increase the comparability of the two populations. Third, all study participants came from a single center. Therefore, readers should be cautious that these results may or may not apply to other populations in other regions. Our findings should be verified and extended in larger, multi-center studies.

\section{Acknowledgements}

This work was supported by the Graduate Course Construction Project of Guangxi Medical University (YJSA2017014), the Self-Raised Scientific Research Fund of the Ministry of Health in Guangxi Province (Z2016512, Z2015621, GZZC15-34, Z2016479), the Foundation Ability Enhancement Project for Young Teachers in Guangxi Universities (2018KY0122), the National Major Special Science and Technology Project (2017ZX10203207), the National Natural Science Foundation of China (81560460/H1602), and 'Guangxi BaGui Scholars' special fund. Funding agencies had no role in study design, data collection and analysis, decision to publish, or preparation of the manuscript.

\section{Author Contributions}

J.-H.Z. conceived the study. Y.-Y.L., R.-R.J., J.C., L.M. and J.-H.Z. collected and analyzed data. Y.-Y.L., and R.-R.J. analyzed data. Y.-Y.L. and J.-H.Z. drafted the manuscript. B.-D.X., L.-Q.L., and N.-F.P. revised 
the manuscript. All authors have read and approved the final version of the manuscript.

\section{Competing Interests}

The authors have declared that no competing interest exists.

\section{References}

1. Bray F, Ferlay J, Soerjomataram I, et al. Global cancer statistics 2018: GLOBOCAN estimates of incidence and mortality worldwide for 36 cancers in 185 countries. CA Cancer J Clin. 2018;68(6):394-424.

2. EASL Clinical Practice Guidelines: Management of hepatocellular carcinoma. J Hepatol. 2018;69(1):182-236.

3. Zhong JH, Peng NF, You XM, et al. Tumor stage and primary treatment of hepatocellular carcinoma at a large tertiary hospital in China: A real-world study. Oncotarget. 2017;8(11):18296-18302.

4. Kim HL, An J, Park JA, et al. Magnetic Resonance Imaging Is Cost-Effective for Hepatocellular Carcinoma Surveillance in High Risk Patients with Cirrhosis. Hepatology. 2019;69(4):1599-1613.

5. Harimoto N, Yoshizumi T, Inokuchi S, et al. Prognostic Significance of Preoperative Controlling Nutritional Status (CONUT) Score in Patients Undergoing Hepatic Resection for Hepatocellular Carcinoma: A Multi-institutional Study. Ann Surg Oncol. 2018;25(11):3316-3323.

6. Takagi K, Umeda Y, Yoshida R, et al. Preoperative Controlling Nutritional Status Score Predicts Mortality after Hepatectomy for Hepatocellular Carcinoma. Dig Surg. 2019;36(3):226-232

7. Goldwasser P, Feldman J. Association of serum albumin and mortality risk. J Clin Epidemiol. 1997;50(6):693-703.

8. Dong ZR, Zou J, Sun D, et al. Preoperative Albumin-Bilirubin Score for Postoperative Solitary Hepatocellular Carcinoma within the Milan Criteria and Child-Pugh A Cirrhosis. J Cancer. 2017;8(18):3862-3867.

9. Kuszajewski ML, Clontz AS. Prealbumin is best for nutritional monitoring. Nursing. 2005;35(5):70-71.

10. Huang J, Wang Y, Yuan Y, et al. Preoperative serum pre-albumin as an independent prognostic indicator in patients with localized upper tract urothelial carcinoma after radical nephroureterectomy. Oncotarget. 2017;8(22):36772-36779.

11. Cavallin F, Scarpa M, Cagol M, et al. Low perioperative serum prealbumin predicts early recurrence after curative pulmonary resection for non-small-cell lung cancer. World J Surg. 2013;37(8):2005.

12. Zhong JH, Xiang BD, Gong WF, et al. Comparison of long-term survival of patients with BCLC stage B hepatocellular carcinoma after liver resection or transarterial chemoembolization. PLoS One. 2013;8(7):e68193.

13. Zhong JH, Ke Y, Gong WF, et al. Hepatic resection associated with good survival for selected patients with intermediate and advanced-stage hepatocellular carcinoma. Ann Surg. 2014;260(2):329-340.

14. Shen Y, Shi G, Huang C, et al. Prediction of Post-Operative Liver Dysfunction by Serum Markers of Liver Fibrosis in Hepatocellular Carcinoma. PLoS One. 2015;10(10):e0140932.

15. Chen X, Zou H, Xiong L, et al. Predictive power of splenic thickness for post-hepatectomy liver failure in HBV-associated hepatocellular carcinoma patients. World J Surg Oncol. 2017;15(1):216.

16. Lu SD, Wang YY, Peng NF, et al. Preoperative Ratio of Neutrophils to Lymphocytes Predicts Postresection Survival in Selected Patients With Early or Intermediate Stage Hepatocellular Carcinoma. Medicine (Baltimore). 2016;95(5):e2722.

17. $\mathrm{Yu} \mathrm{JJ}$, Shen $\mathrm{F}, \mathrm{Chen} \mathrm{TH}$, et al. Multicentre study of the prognostic impact of preoperative bodyweight on long-term prognosis of hepatocellular carcinoma. Br J Surg. 2019;106(3):276-285. .

18. Zhao LY, Huo RR, Xiang $X$, et al. Hepatic resection for elderly patients with hepatocellular carcinoma: a systematic review of more than 17,000 patients. Expert Rev Gastroenterol Hepatol. 2018;12(10):1059-1068.

19. Wang YY, Zhong JH, Su ZY, et al. Albumin-bilirubin versus Child-Pugh score as a predictor of outcome after liver resection for hepatocellular carcinoma. $\mathrm{Br}$ J Surg. 2016;103(6):725-734.

20. Levitt DG, Levitt MD. Human serum albumin homeostasis: a new look at the roles of synthesis, catabolism, renal and gastrointestinal excretion, and the clinical value of serum albumin measurements. Int J Gen Med. 2016;9:229-255.

21. Shenkin A. Serum prealbumin: Is it a marker of nutritional status or of risk of malnutrition? Clin Chem. 2006;52(12):2177-2179.

22. Beck FK, Rosenthal TC. Prealbumin: A marker for nutritional evaluation. Am Fam Physician. 2002;65(8):1575-1578.

23. Bae HJ, Lee HJ, Han DS, et al. Prealbumin Levels as a Useful Marker for Predicting Infectious Complications After Gastric Surgery. J Gastrointest Surg. 2011;15(12):2136-2144

24. Fujii T, Sutoh T, Morita H, et al. Serum Albumin Is Superior to Prealbumin for Predicting Short-Term Recurrence in Patients with Operable Colorectal Cancer. Nutr Cancer. 2012;64(8):1169-1173.
25. Kawai $\mathrm{H}$, Ota $\mathrm{H}$. Low perioperative serum prealbumin predicts early recurrence after curative pulmonary resection for non-small-cell lung cancer. World J Surg. 2012;36(12):2853-2857.

26. Huang L, Li J, Yan JJ, et al. Prealbumin is predictive for postoperative liver insufficiency in patients undergoing liver resection. World J Gastroenterol. 2012;18(47):7021-7025.

27. Jia RR, Zhong JH, Huo RR, et al. Correlation between serum prealbumin and prognosis of patients with hepatocellular carcinoma after hepatectomy. J Surg Oncol. 2019;119(6):794-800.

28. Li JD, Xu XF, Han J, et al. Preoperative prealbumin level as an independent predictor of long-term prognosis after liver resection for hepatocellular carcinoma: a multi-institutional study. HPB (Oxford). 2019;21(2):157-166.

29. Zhao WC, Zhang HB, Yang N, et al. Preoperative predictors of short-term survival after hepatectomy for multinodular hepatocellular carcinoma. World J Gastroenterol. 2012;18(25):3272-3281. 